\title{
Demonstration of a Multiplex Milk Allergen ELISA using Oligonucleotide-tethered Principal Component Proteins
}

\author{
Robert S. Matson* \\ Robert S. Matson, QuantiScientifics LLC, 1920 E. Katella Ave. Suite S, Orange, CA 92867, USA
}

Received: 23 February, 2016; Accepted: 25 March, 2016; Published: 27 March, 2016

*Corresponding author: Robert S. Matson, QuantiScientifics LLC, 1920 E. Katella Ave. Suite S, Orange, CA 92867, USA, Tel : +1 714-624-0810; E-mail: rsmatson@quantiscientifics.com

\begin{abstract}
The A-Squared $\left(\mathrm{A}^{2 \circledast}\right)$ Microarray System (QuantiScientifics, Orange, CA, USA) is a micro plate-based, microarray platform for quantitative multiplexed immunoassays. Arrays of capture oligonucleotides are covalently attached to the bottom of the plate well. Complementary strands are in turn linked to proteins such as antibodies or antigens. The resulting Oligo-Antibody (-Antigen) conjugates are pooled and applied to the wells. Through the process of hybridization, capture oligo-oligo protein hybrid pairs undergo self-assembly to form an "Oligonucleotide-Tethered" antibody or antigen array in each well.

In this study, we demonstrate the ability to tether known "Allergenic" proteins found in cow's milk to create a multiplex immunoassay useful in the diagnosis of Serological (IgE-Mediated) cow's milk allergy. Purified milk proteins whose epitopes are known to be associated with the allergy, as well as, crude milk allergen extracts were conjugated to oligonucleotides to create the panel. The panel included F2 crude milk protein extract, as well as, principal component proteins Beta-Lacto Globulin, alpha-lactalbumin, IgG, Bovine Serum Albumin, Lactoferrin, and 3 caseins. Patient serums were screened for the presence of specific IgE (sIgE) providing a profile and subsequent identification of principal component allergen.

Keywords: Milk; F2; Allergen; Multiplex; Microarray; Micro plate; ELISA; Fluorescence; Immunoassay; Oligonucleotide; Tethered; Conjugation; Self-Assembly; Hybridization; Principal Component; Specific Ige; Crude Extract; $\mathrm{A}^{2}$ Microarray System
\end{abstract}

\section{Introduction}

Microarrays, as a multiplex immunoassay platform, have been used in the assessment of allergen specific-IgE (sIgE) for over the past 15 years. Wiltshire, et al. [1] prepared extracts of cat hair, dust mites and peanut, and then arrayed these onto an activated glass slide (microarray substrate). Sera with known levels of sIgE based upon Auto Cap (Pharmacia) values were applied. Detection of the sIgE on the microarrays was accomplished using ImmunoRCA (Rolling Circle Amplification) and the results compared to the CAP values. The microarray method was found to have a higher positive predictive value, higher specificity and in most cases greater sensitivity. Likewise, Kim, et al. [2] dispensed prepared extracts of various allergens onto a nitrocellulose slide (FAST Slide, Schleicher \& Schull). The presence of sIgE In sera For Allergens (Dermatophagoides pteronyssinus (Dp), Egg White, Soybean, Milk, and Wheat) was determined by immune fluorescence laser scanning of the microarray. The microarray results correlated well with those determined using UniCap (Pharmacia \& Upjohn Diagnostics AB).

Bacarese-Hamilton, [3] prepared allergen microarrays from extracts onto activated glass slides and evaluated sensitivity and specificity using the Tyramide Signal Amplification (TSA) catalyzed by HRP (Molecular Probes). A lower limit of detection of the microarray bound $\operatorname{sgE}<1 \mathrm{fg}$ was achieved from $100 \mu \mathrm{l}$ sera corresponding to about $10 \mathrm{fg} / \mathrm{mL}$ equivalent to $\sim 4.1 \times 10^{-6}$ $\mathrm{IU} / \mathrm{mL}(1 \mathrm{IU} / \mathrm{mL} \operatorname{IgE}=2.44 \mathrm{ng} / \mathrm{mL})$ or a cut-off value at $4.1 \mathrm{k}$ $\mathrm{IU} / \mathrm{L}$.

Lebrun, [4] adapted the standard ELISA for quantitative determinations on a nitrocellulose substrate (Zeta-Grip, Miragene) using a flat-bed scanner for colorimetric detection of the developed microarray. Sensitivities for sIgE were achieved that fell below the World Health Organization (WHO) cut-off value of $0.35 \mathrm{k} \mathrm{IU} / \mathrm{L}$.

Hiller, et al. [5] first immobilized purified or recombinant allergenic proteins (rather than crude extracts) onto amine reactive glass slides (VBC-Genomics, Vienna) to measure allergen sIgE. This approach subsequently led to the co-development with Phadia of the ISAC (Immuno Solid-phase Allergen Chip) microarray ushering in Component-Resolved Diagnostic (CRD) for allergen testing. The ImmunoCAP ISAC chip products are commercially available from Thermo Fisher Scientific for the assessment of sIgE.

Ott, et al. [6] used purified allergenic proteins from milk and eggs spotted onto a microarray slide in an effort to assess the clinical predictive value of component resolved diagnosis. The study encompassed 130 children with allergy to milk or eggs and included correlation of the microarray to that of UniCap (Phadia). The authors concluded, " that microarray-based IgE 
quantification was accurate in predicting clinical reactivity to allergenic proteins in our study population".

Martinez-Aranguren, et al. [7] evaluated the ImmunoCAP ISAC 112 chip kit comprising 5 slides with each slide containing 4 sub-arrays of 112 protein components covering 51 allergens. The validation study was based upon manufacturer's calibrators, as well as, sera obtained from 19 patients and included inter-assay, intra-assay and inter-lab analyses. The mean Coefficient of Variation (CV) for calibrators was $9.42 \%$. The Intra-Class Coefficient Correlation (ICC) was used as a measure of reproducibility and repeatability. For inter-assay (reproducibility), determinations for IgE in 94/112 (83.9\% of allergens) had very good reproducibility (ICC $>0.90$ ), as was found for the reproducibility (intra-assay) of this set. The inter-laboratory agreement was very good for $73 / 112$ allergens (ICC $>0.90)$ and good for 22 (ICC $=0.71-0.90)$. While the microarray performed well, there were several instances noted in which the expected values were not achieved leading the authors to conclude that, "due to the low accuracy obtained in some (reported 7-8 components) of the studied allergens, the application of this semi-quantitative technique for diagnosis in clinical situations where results may have a major impact on the therapy prescribed may not be advisable".

However, Moreira, et al. [8] evaluated sera of patients from Brazil having grass pollen allergy using ImmunoCAP ISAC microarrays. In their study, an ISAC chip containing 103 components was used that included 9 allergens for timothy grass pollen and 1 allergen for Bermuda grass. In this case, 77 of 78 patients tested positive for timothy grass pollen. Thus, these researchers suggest that the ISAC allergen microarray could be useful in diagnosis and immunotherapy monitoring for patients suffering from Brazilian grass pollens.

Studies on the development of microarrays in general and specifically for allergen testing have been largely based upon surface activated glass or nitrocellulose coated glass slide substrates. Micro plate-based microarray platforms are now commercially available and being adopted for multiplex immunoassays including allergy testing. The $\mathrm{A}^{2}$ Microarray System (QuantiScientifics) is a multiplex quantitative immunoassay platform based upon a 96-well polypropylene plate to which proteins maybe tethered via an oligonucleotide bridge to create antibody or antigen microarrays. For example, Robbins, et al. [9] created an $\mathrm{A}^{2}$ plate cytokine antibody array in order to measure the release of cytokines resulting from the immuno-stimulatory effects of lipid-delivered siRNAs in primary CD34+progenitor-derived hematopoietic cells.

Here, we examine the utility of the $A^{2}$ multiplex assay platform in the development of component-resolved diagnostics for allergens based upon oligonucleotide tethering of allergenic proteins. In these studies, we use both purified milk protein allergens and crude extracts as a model [10].

\section{Materials and Methods}

The following reagents were obtained from QuantiScientifics
(Orange, CA) and used according to the manufacture's kit instructions:

\section{A2 Capture Oligo Plate (A21002)}

Enzyme Linked Assay Kit (A21003) containing Hybridization Buffer (A21003-HYB), Biotinylated Reference Oligo (A21003REF), streptavidin alkaline phosphatase, AP Enzyme Substrate (A21003-SUB), wash buffer; Conjugation Kit: oligo-antibody coupling (A21004) containing spin-columns, solid-phase (ButylSepharose), initiator (iminothiolane), binding \& elution buffers.

Binding Buffer: sodium phosphate, $20 \mathrm{mM}$ containing sodium sulfate, $1 \mathrm{M}, \mathrm{pH} 7.5$.

Elution Buffer: sodium phosphate, 20mM, pH 7.5.

\section{Activated oligonucleotides}

4-(N-Maleimido methyl) cyclohexane-1-carboxylic acid N-hydroxy succinimidyl-terminated oligonucleotides (A21005QS1, -QS3, -QS5, -QS9, -QS13 \& -QS17). Oligonucleotides are complementary to the microarray capture oligos covalently attached to the A2 Capture Oligo Plate. Length, 30mer; Melt Temperature, $\mathrm{Tm} \sim 64^{\circ} \mathrm{C}$.

Wash buffer: Tris Buffered Saline with Tween 20, pH 8.0 (TBST: Sigma-Aldrich, T9039), 0.05M Tris, 0.138M NaCl, 0.0027M $\mathrm{KCl}$, Tween 20, 0.05\%.

A2 microarray reader (A21001): Optical Cube A installed, Ex $545 \mathrm{~nm}$, Em $650 \mathrm{~nm}$ and Optical Cube B installed, Ex $480 \mathrm{~nm}$, Em $535 \mathrm{~nm}$

\section{Proteins}

Cow's milk derived proteins were obtained in purified form from Sigma-Aldrich (see Table 1). Streptavidin-alkaline phosphates conjugate (Thermo Scientific, Pierce \# 21324); Goat anti-human IgE alkaline phosphatase conjugates (Sigma-Aldrich, A3525).

Serum Samples: Hycor Biomedical (Garden Grove, CA) provided milk allergenic positive patient sera and spiked control sera for evaluation.

\section{Preparation of oligonucleotide-protein conjugates}

$20 D_{260 \mathrm{~nm}}$ units of an SMCC (4-(N-maleimidomethyl) cyclohexane-1-carboxylic acid N-hydroxysuccinimidyl) activated oligonucleotide are reacted with $100 \mu$ g of purified protein. The process involves adsorption (hydrophobic interaction) of the protein onto a solid-phase held within a spin-column. Next, initiator is added to modify the protein with reactive sulfhydryl $(-\mathrm{SH})$ groups. Finally, the SMCC-oligonucleotide is added which covalently couples to the thiolate protein to form the oligoprotein conjugate. The resulting conjugate still bound to the solid-support is rinsed free of reactants; and subsequently eluted in purified form from the column ready for use.

Preparation of oligo- F2 (1) $\beta$-Lacto globulin conjugate from purified protein

Protein modification: $100 \mu \mathrm{L}$ Butyl-Sepharose 4 Fast Flow 
Table 1: Description of Principal Protein Components.

\begin{tabular}{|l|l|l|l|l|}
\hline $\begin{array}{l}\text { Comp } \\
\#\end{array}$ & Component & $\begin{array}{l}\text { Molecular } \\
\text { Weight }\end{array}$ & Source & $\begin{array}{l}\text { SMCC- } \\
\text { Oligo }\end{array}$ \\
\hline F2 & F2 crude extract & NA & Hycor & QS17 \\
\hline F2-1 & $\beta$-lactoglobulin & 18,300 & L3908 & QS1 \\
\hline F2-2 & $\alpha$-lactalbumin & 14,200 & L6010 & QS3 \\
\hline F2-3 & IgG & 150,000 & I5506 & QS5 \\
\hline F2-4 & $\begin{array}{l}\text { Bovine Serum } \\
\text { Albumin (BSA })\end{array}$ & 66,300 & A9085 & QS9 \\
\hline F2-5 & Lactoferrin & 80,000 & L9507 & QS1 \\
\hline F2-6 & $\alpha$ S-casein & 25,000 & C6780 & QS3 \\
\hline F2-7 & $\beta$-casein & 24,000 & L6905 & QS5 \\
\hline F2-8 & r-casein & 10,000 & L0406 & QS9 \\
\hline & & & $\begin{array}{l}\text { Immuno } \\
\text { Reagents } \\
\text { GtxHu-002-D }\end{array}$ & QS13 \\
\hline & Goat anti-IgE & 150,000 & & Win
\end{tabular}

List of known allergenic proteins associated with milk allergy used in this study.

Comp\# (Milk Allergen Principal Component nomenclature for proteins used in this study) Molecular Weight (Daltons), Source (Sigma-Aldrich catalog number unless otherwise stated), SMCC-oligo assignments QS1,3,5,9,13,17 refer to unique sequences of SMCC terminated oligo nucleotides for covalent coupling to specified component proteins.

(GE Healthcare Bio-Sciences AB, Uppsala, Sweden) was placed in a fritted spin column to achieve a compressed bed volume of 0.1 $\mathrm{mL}$. The column was centrifuged at $2000 \mathrm{rpm}$ for 30 seconds to remove liquid.

The resin was re-suspended in $600 \mu \mathrm{L}$ of deionized water using a pipette; and the column washed by centrifugation. The centrifugation-rinse process was repeated, followed by 2 rinses in binding buffer.

Following the equilibration in binding buffer, the bottom of the column was carefully sealed with a cap.

$600 \mu \mathrm{L}$ of binding buffer was used to re-suspend the resin. Then, $50 \mu \mathrm{L}=100 \mu \mathrm{g}$ of $\beta$-Lacto globulin (Sigma-Aldrich, L3908, MW 18,300 Daltons) from stock solution of $2 \mathrm{mg} / \mathrm{mL}$ in TBST buffer was added. The resin was then mixed by Pipetting up \& down and column recapped.

Following end-to-end tumbling of the column for 10 minutes, the caps were removed and the column centrifuged to remove the solution.

$700 \mu \mathrm{L}$ of freshly prepared protein activator solution (Iminothiolane Hydrochloride, Sigma-Aldrich, 16256) was added to the column bed; and the resin re-suspended. The column was capped and then mixed by tumbling for 1 hour.

The caps were then removed and the column centrifuged and the bed re-suspended, then rinsed 6 times with binding buffer as previously described.

Finally, $600 \mu \mathrm{L}$ of fresh binding buffer was added, the column bottom capped and the resin re-suspended.

\section{Coupling of the oligonucleotide to thiol-protein}

$100 \mu \mathrm{L}$ of SMCC-oligo (2 ODs) prepared in binding buffer was added and the resin re-suspended and column capped. The spincolumn was mixed by tumbling for 24 hours at room temperature.

Following removal of the solution, the column was rinsed 5 times in binding buffer in order to remove reactants.

\section{Elution of the oligo-protein conjugate}

The column was then placed in a clean 1.5-mL Eppendorf micro-centrifuge tube for collection of the conjugate.

$100 \mu \mathrm{L}$ of elution buffer was added to the column with gentle mixing to re-suspend the resin. The column was incubated for 3 minutes and then centrifuged. The eluted fraction was collected as the source of the conjugate.

This process was repeated 4 more times with the collection of $100 \mu \mathrm{L}$ fractions in separate tubes for a total of 5 fractions (500 $\mu \mathrm{L})$.

The eluted fractions were combined and the solution stored at $4^{\circ} \mathrm{C}$ prior to use.

Preparation of the oligo-F2 (2); -F2 (3); -F2 (4); -F2 (5); -F2 (6); -F2 (7) and -F2 (8) conjugates

Additional milk proteins, F2 crude extract, as well as, the goat anti-Human IgE were conjugated to SMCC-oligonucleotides as described in the previous section detailing the preparation of oligo-F2 (1) conjugate. The assignments regarding choice of oligo used in coupling are provided in Table 1 . The selected Placement

\section{Array Map I}

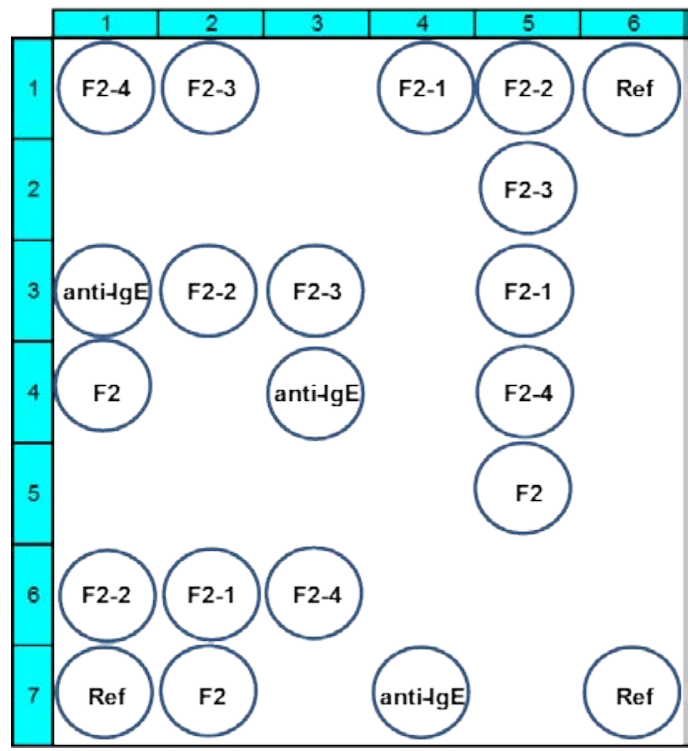

Figure 1: Array Format I

Array I well map for placement of microarray spots in each well corresponding to the various hybridized oligo-proteins listed in Table 1. 


\begin{tabular}{|c|c|c|c|c|c|c|c|c|c|c|c|c|c|c|}
\hline F2\# & Protein & S1 & S2 & S3 & S4 & S5 & S6 & S7 & S8 & S9 & S10 & S11 & S12 & S13 \\
\hline 1 & $\beta$-lactoglobulin & & 0.428 & & 0.062 & & 2.348 & & & 0.698 & & & 2.458 & 0.326 \\
\hline 2 & $\alpha$ - lactalbumin & & 1.174 & & & & 1.373 & & & 0.258 & & & 0.951 & 0.064 \\
\hline 3 & $\operatorname{IgG}$ & 0.206 & & & & & 1.123 & & & & & & & 0.244 \\
\hline 4 & BSA & & & & & & 3.164 & & & 0.127 & & 0.364 & & 0.459 \\
\hline 5 & Lactoferrin & 0.525 & & & & & 0.082 & & & & & & & \\
\hline 6 & $\alpha \mathrm{S}$-casein & & 0.611 & & 0.688 & & & & & 0.725 & & & 0.627 & 0.442 \\
\hline 7 & $\beta$-casein & & 0.098 & & & & & & & & & & & 0.911 \\
\hline 8 & r-casein & & 0.154 & & 0.397 & & 0.454 & & & & & & & 1.133 \\
\hline
\end{tabular}

Component specific IgE (sIgE) in serum samples (S1 to S13) determined using the $\mathrm{A}^{2}$ Multiplex Immunoassay.

Mean Fluorescent Intensity (MFI) signal values obtained from the microarray spots were converted to International Units (IU) based upon the IgE standard curve (MFI vs. IU/ mL) provided in Figure 3. Values $\leq 0.35 \mathrm{IU} / \mathrm{mL}$ are reported for indication only as a Minimal Detectable Dose (MDD).

\section{Array Map II}

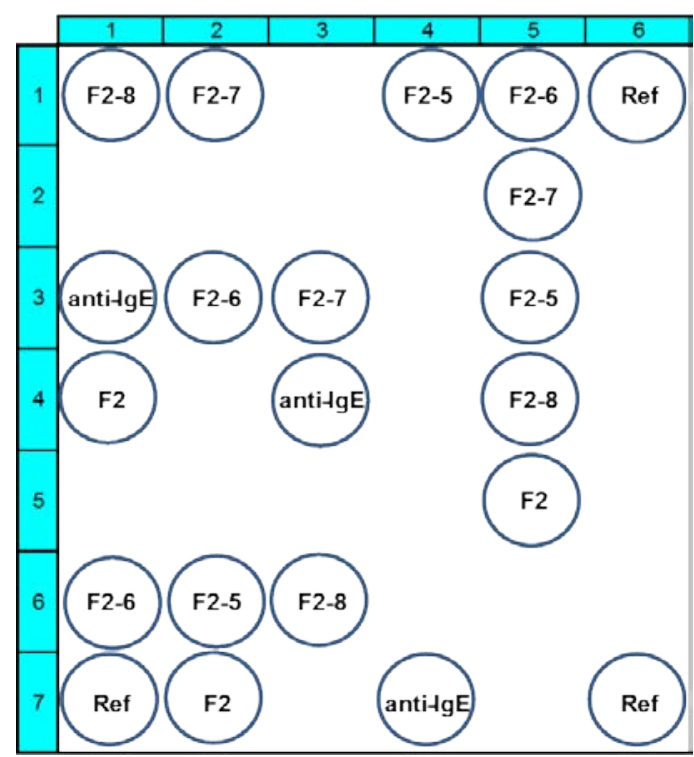

Figure 2: Array Format II

Array II well map for placement of microarray spots in each well corresponding to the various hybridized oligo-proteins listed in Table 1.

within the array well are for format I (Figure 1) or array format II (Figure 2).

\section{Preparation of the microplate microarray with oligonucleotide-tethered milk allergens}

The oligo-protein conjugates were pooled in hybridization buffer at $2 \mu \mathrm{g} / \mathrm{mL}$ of each for application to the A2 capture oligo plate. Following a 5 minute soak and rinse of the plate wells with $3 \times 200 \mu \mathrm{L}$ TBST, $55 \mu \mathrm{L}$ of the oligo-protein hybridization cocktail was delivered to each well using a pipette. The plate was incubated at ambient temperature with shaking for 1 hour. The wells were rinsed 3-times in TBST in preparation of the immunoassay.

\section{Development of the multiplex allergen immunoassay}

Serum samples (1-13) containing sIgE, negative control serum without IgE, and reference control sera containing varying amounts of $\operatorname{IgE}(0.35 \mathrm{IU} / \mathrm{mL}$ to $100 \mathrm{IU} / \mathrm{mL})$ as standards (1 IU $=2.44 \mathrm{ng}$ ), as well as, the biotinylated reference oligo (fiducial) were delivered to wells of the microplate at $55 \mu \mathrm{L}$ per well. The plate was incubated for 1 hour with shaking. Following TBST buffer rinse, a secondary antibody-alkaline phosphates conjugate (goat anti-human IgE) was applied in buffer at $55 \mu \mathrm{L}$ per well. Reference fiducials of the biotinylated oligo were detected by the addition of streptavidin alkaline phosphatase to the mix. The plate was incubated for 1 hour with shaking, and then extensively rinsed in buffer. Next, $55 \mu \mathrm{L}$ of AP Enzyme Substrate was added to each well. The plate was covered with foil and incubated for 30 minutes without shaking. The solution was carefully removed to semi-dry but not rinsed. $200 \mu \mathrm{L}$ of fresh buffer was added to the developed plate and the plate scanned using the A2 Reader. Note: the proprietary enzyme substrate forms a fluorescent precipitate (similar to the tyramide signal amplification process) which is detected at $535 \mathrm{~nm}$.

\section{Analysis}

An ELISA standard curve (Figure 3, 4PL2 plot, AssayFit, IVDtools, Inc. Nijmegen, The Netherlands) was generated from serial dilution of the reference control sera. Fitted Curve:Response (MFI, mean fluorescent intensity) vs. Dose (Human IgE, IU/ mL). The IgE content in serum samples, negative serum and buffer was calculated from the curve. The $\mathrm{A}^{2}$ Microarray System reader reports in $\mathrm{pg} / \mathrm{mL}$ units. Conversion to International Units (IU) was made based upon: $1 \mathrm{IU} / \mathrm{mL} \mathrm{IgE}=2.44 \mathrm{ng} / \mathrm{mL}=2440 \mathrm{pg}$ / $\mathrm{mL}$.

\section{Results}

The total IgE as measured from signal on arrayed spots (Figure 4) containing oligo-gt anti IgE was comparable across both well array formats $(r=0.9764)$, Figure 5 .

Likewise, signal (MFI) corresponding to sIgE was obtained for all samples. Negative control serum (MFI = $337 \pm 67$ ) and buffer blank (MFI= $259 \pm 53$ ) values were used to establish a cut- 


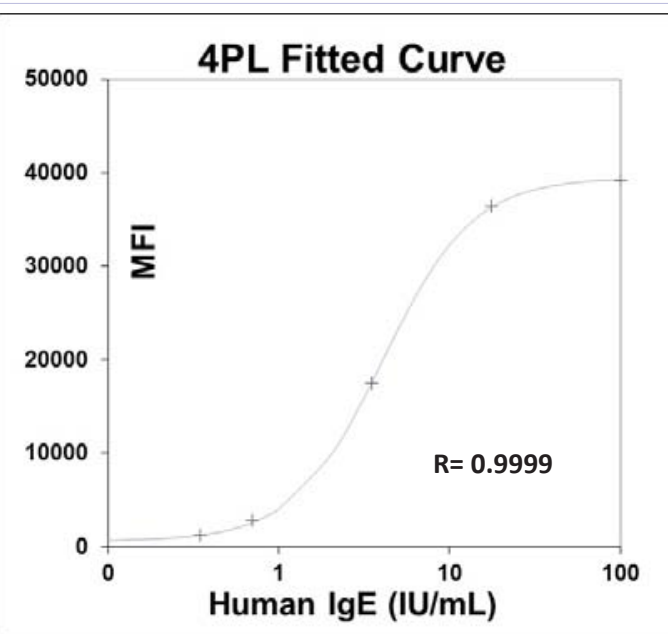

Figure 3: IgE ELISA Standard Curve

dose-response 4PL plot for estimating the IgE content in the serum samples.

4PL (4 Parameter Logistic nonlinear regression model).MFI (mean fluorescence intensity). Range: $0.35-100 \mathrm{IU} / \mathrm{mL}$. MDD (minimal detectable dose) $\leq 0.35 \mathrm{IU} / \mathrm{mL}$. The A2 MicroArray System reports in $\mathrm{pg} / \mathrm{mL}$. Conversion to $\mathrm{IU} / \mathrm{mL}$ is based upon $1 \mathrm{IU} / \mathrm{mL} \operatorname{IgE}=2440 \mathrm{pg} / \mathrm{mL}$.

\section{Detection of serum IgE in well array}

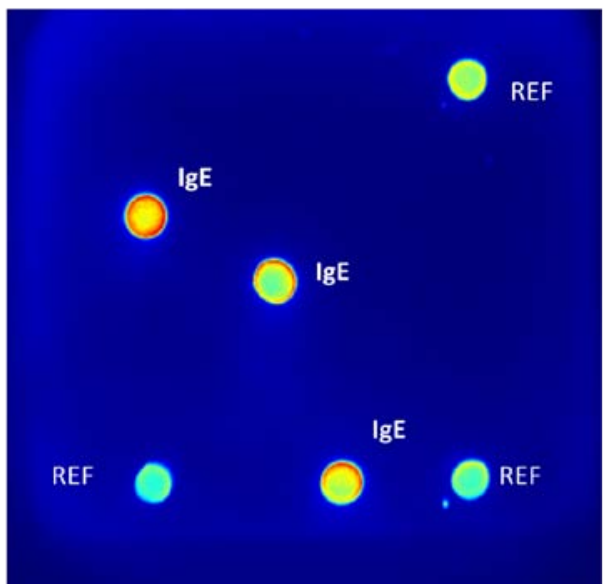

Figure 4: screen capture of an A2 Plate well image from the A2 MicroArray System.

REF (reference: biotinylated oligo). IgE (oligo-goat anti IgE capture antibody). Signal developed using streptavidin-alkaline phosphatase conjugate (REF signal) + goat anti-Hu IgE alkaline phosphatase (IgE signal) with a proprietary fluorescent substrate that forms a precipitant detectable at $535 \mathrm{~nm}$.

off baseline for non-specific binding at MFI = 538 corresponding to the negative control, MFI+3SD. In this study, the lowest IgE control used was $0.35 \mathrm{IU} / \mathrm{mL}$ yielding a signal, MFI = 1249 or about 2.3-fold above the cut-off value. Using the baseline cutoff, the identity and level of the principal "allergenic" component present in each sample could be more easily recognized in a composite profile (Figure 6).
Array I wells detected the presence or absence of sIgE in sera for immunological response to cow's milk proteins, principal components: F2-1 ( $\beta$-lactoglobulin), F2-2 ( $\alpha$-lactalbumin), immunoglobulin G (IgG), and Bovine Serum Albumin (BSA). Spots containing immobilized goat anti-human IgE were used to estimate total IgE content in the sample. Very high levels (> $200,000 \mathrm{pg} / \mathrm{mL}$ or $200 \mathrm{ng} / \mathrm{mL} \sim 82 \mathrm{IU} / \mathrm{mL}$ ) of total IgE were found in samples \#3, \#7, \#9 and \#11. These particular samples exhibited very low or non-detectable levels of sIgE (Figure 7). In comparison, samples \#6 and \#12, while exhibiting low total IgE levels were found to contain mostly sIgE. Sample \#6 contained sIgE toward F2-1, F2-2, F2-3 and F2-4; while sample \#12 was prominent for F2-1(Figure 8, $\beta$-lactoglobulin) and F2-2.

From Array II, sample \#1 was predominate for F2-5 (Figure 9 , lactoferrin), while sample \#13 contained modest amounts of sIgE for F2-7 ( $\beta$-casein) and F2-8 (r-casein). Samples \#2, \#6, \#9, $\# 12$ and \#13 had detectable levels of F2-6 ( $\alpha$ S-casein) above background. Coupling of oligonucleotide (QS17) to a crude milk protein extract was achieved providing the signal profiling sIgE's in serum samples but with a lowered response (Figure 10).

A summary of the estimated content of specific $\operatorname{IgE}$ in the patient serum samples in provided in Table 2. Values $\leq 0.35 \mathrm{IU} /$

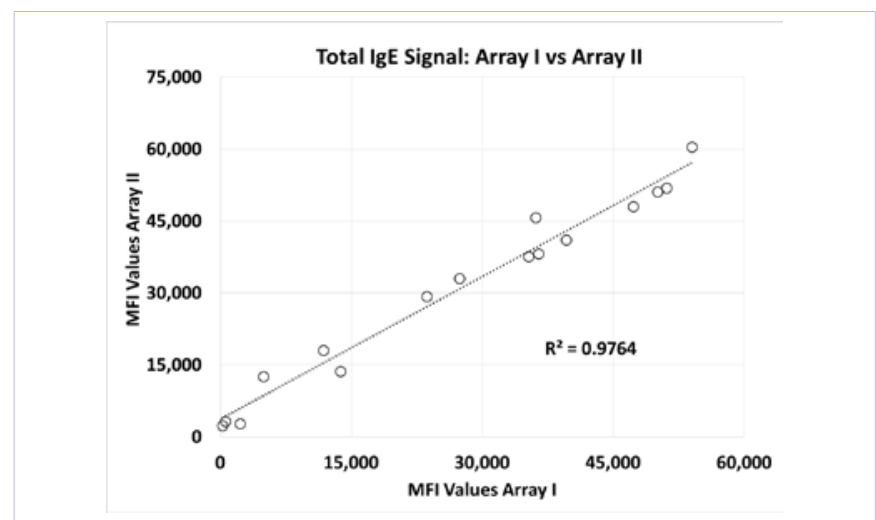

Figure 5: Correlation for Total IgE Signal between Arrays Correlation between array I and array II signal intensity obtained from microarray spots at various concentrations of IgE standard input.

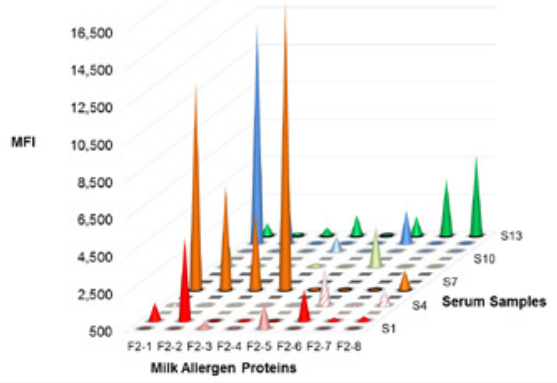

Figure 6: Milk Allergen Principal Components Profiles in Patient Serum Identification of major allergenic milk proteins associated with the IgE levels found in serum samples. 
$\mathrm{mL}$ are the result of extrapolation below the lowest standard and should be regarded for indication only.

\section{Discussion}

The $\mathrm{A}^{2}$ Microarray technology is an open platform based upon a pre-printed and fixed pattern (microarray) of capture oligonucleotides in a 96-well micro plate, the $\mathrm{A}^{2}$ Plate. Multiplex ELISAs once designed by the end-user are constructed using the complementary oligo linked to the selected proteins. A mixture of different oligo-proteins may then be hybridized to the microarray to create a protein array. For example, the tethering of oligo-antibody to form an antibody array is the primary application for this technology platform. Since, hybridization is a thermodynamically driven; self-assembly process the number of oligo/oligo-protein hybrids formed on the surface is limited and relatively constant. This results in improved spot uniformity within the array and reduces the variability commonly found with direct printing of proteins. Moreover, the oligo-protein conjugates are reagents that can be stored separately and combined as needed to create different plate assays upon demand.

Allergens represent a complex mixture of proteins and other biomolecules. It is most often difficult to resolve those proteins that are associated with a particular "allergenic" response.

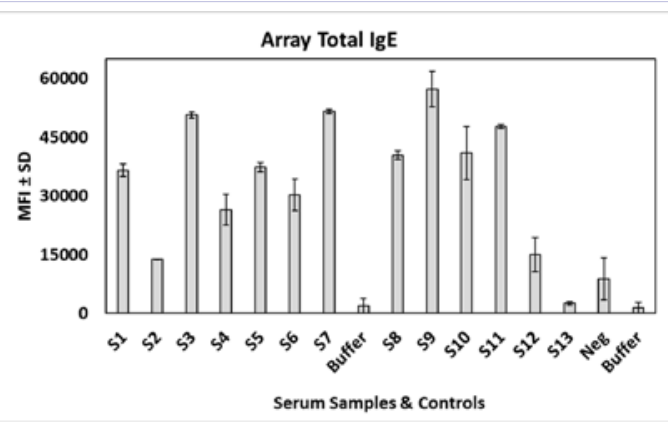

Figure 7: Total IgE in Samples

Total IgE profile determined from QS13 anti-human IgE signal. Mean Fluorescence Intensity (MFI) from array I \& array II spots. Standard Deviation (SD) for $\mathrm{n}=2$ determinations.

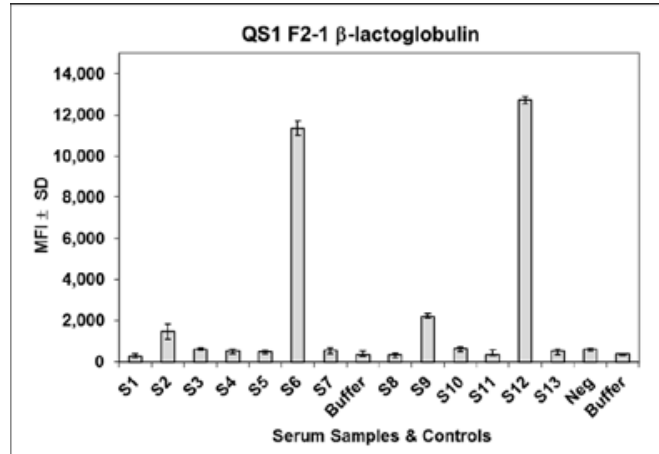

Figure 8: Array Profile for F2-1

sIgE serum profiles determined from QS1oligo tethered $\beta$-lactoglobulin. Mean Fluorescence Intensity (MFI) from array I spots. Standard Deviation (SD) for $\mathrm{n}=2$ determinations.

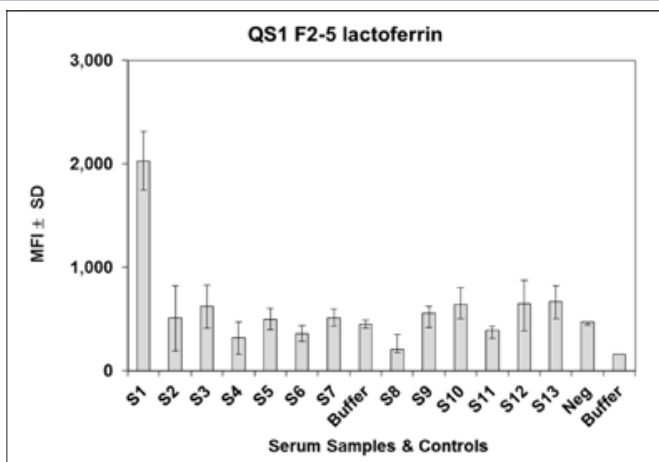

Figure 9: Array Profile for F2-5

sIgE serum profiles determined from QS1 oligo tethered lactoferrin Mean Fluorescence Intensity (MFI) from array I spots. Standard Deviation (SD) for $\mathrm{n}=2$ determinations.

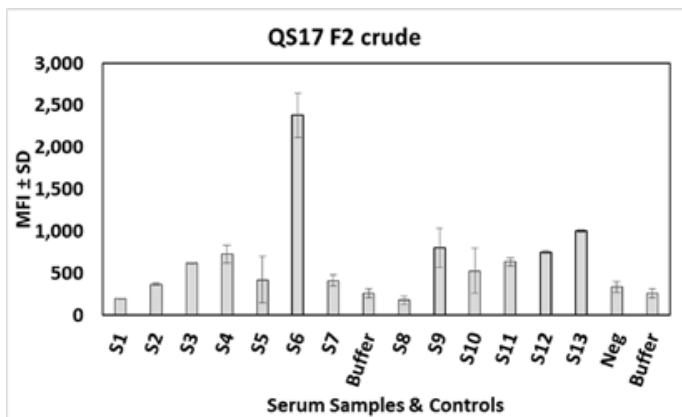

Figure 10: Array Profile for F2 Crude Extract

sIgE serum profiles determined from QS17 oligo tethered F2 crude protein extract.

Mean Fluorescence Intensity (MFI) from array II spots. Standard Deviation (SD) for $\mathrm{n}=2$ determinations.

Because of such complexity, as well as, the vast number of allergens present in our environment it remains an arduous task in diagnosis. Thus, the well- known skin patch test based upon the use of allergen extracts is still prevalent even though a migration and adoption of the immunoassay has been underway for several decades. As previously discussed in the introduction, the development of immunoassays has progressed from e.g. the use of the Radio Allegro Sorbent Test (RAST), to performing a sIgE ELISA using slides or micro plates of printed extracts, to the use of automated diagnostic platforms such as ImmunoCAP or the ImmunoCAP ISAC for component resolved diagnostic platforms that were developed by Phadia (now part of Thermo Fisher Scientific).

In this study, we examined the potential use of the $\mathrm{A}^{2}$ multiplex ELISA in the development of a component resolved methodology for detection of food-based allergens based upon determination of sIgE. Milk allergy was selected as a model because the allergy is prevalent, and the component proteins are readily available in purified form from commercial sources.

The oligo-protein conjugates were successfully prepared, assembled onto the microarray and the IgE ELISA demonstrated. The $\mathrm{A}^{2}$ multiplex ELISA was used to quantify both total IgE and 

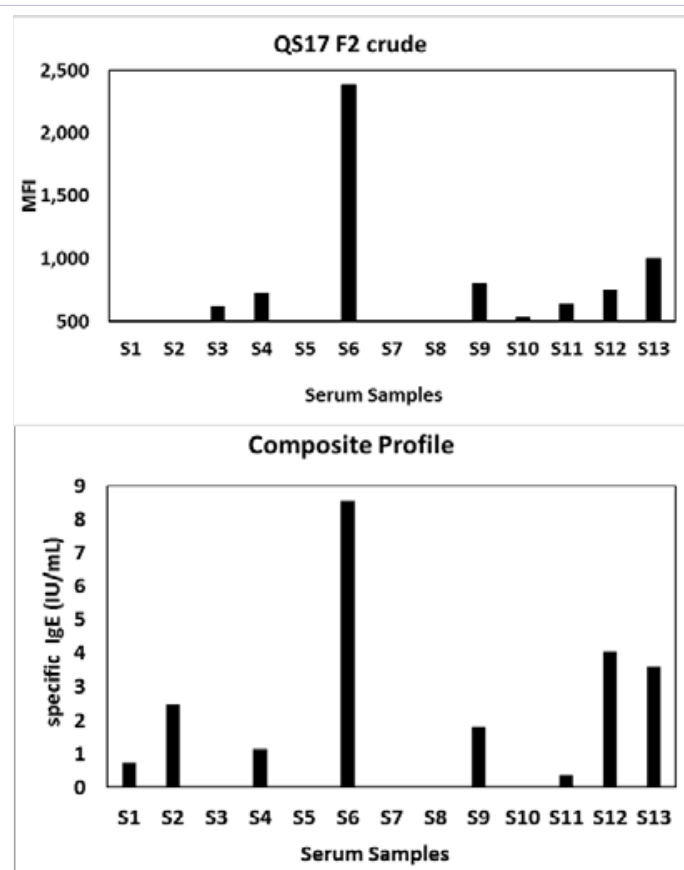

Figure 11: Comparison of sIgE profiles

comparison of sIgE profiles for serum samples based upon reconstruction of a composite profile from calculated component IgE content (Table 2) to that obtained using a milk allergen crude extract (Figure10).

SIgE in serum samples over the range of $0.35-100 \mathrm{IU} / \mathrm{mL}$ In addition to evaluating conjugates of component proteins, the study included a conjugate prepared from the crude milk allergen extract. A profile (Figure 10) of milk allergen content (sIgE) across the serum samples was obtained, albeit at reduced signal intensity relative to individual component proteins. Moreover, it was possible to partially reconstruct the profile (Figure 11) by summation of the sIgE content (IU/ $\mathrm{mL}$ ) of the major component proteins found in each sample listed in Table 2. This provides a further validation that the observed IgE levels detected using the oligo-tethered crude extract reflect the relative contribution of the individual component protein IgE response within the sample.

In summary, this model study demonstrates that oligonucleotide-tethering of proteins of broad molecular weight range (10,000 to 150,000 Daltons) and diverse physical-chemical properties are possible using the $\mathrm{A}^{2}$ technology. Moreover, the microarray bound oligo-allergens were found to be accessible to interaction with serum sIgE allowing the development of a multiplex immunoassay for milk allergens. The A2 multiplex
ELISA permitted screening for sIgE using an oligo-tethered milk allergen extract, as well as, quantitation of component specific IgEand total IgE content of the sample within the same well.

\section{Acknowledgement}

Thanks to Dr. Jang B. Rampal for assistance with experiments; and Hycor Biomedical for providing milk allergen standards and serum samples for this study.

\section{References}

1. Wiltshire S, O’Malley S, Lambert J, Kukanskis K, Edgar D, Kingsmore $\mathrm{SF}$, et al. Detection of multiple allergen-specific IgEs on microarrays by immunoassay with rolling circle amplification. Clin Chem. 2000;46(12):1990-3.

2. Kim TE, Park SW, Cho NY, Choi SY, Yong TS, Nahm BH, et al. Quantitative measurement of serum allergen-specific IgE on protein chip. Exp Mol Med. 2002;34(2):152-8.

3. Bacarese-Hamilton T, Mezzasoma L, Ingham C, Ardizzoni A, Rossi R, Bistoni $\mathrm{F}$, et al. Detection of allergen-specific IgE on microarrays by use of signal amplification techniques. Clin Chem. 2002;48(8):136770.

4. Lebrun SJ, Petchpud WN, Hui A, McLaughlin CS. Development of a sensitive, colorometric microarray assay for allergen-responsive human IgE. J Immunol Methods. 2005;300(1-2):24-31.

5. Hiller R, Laffer S, Harwanegg C, Huber M, Schmidt WM, Twardosz A, et al. Microarrayed allergen molecules: diagnostic gatekeepers for allergy treatment, FASEB J. 2002;16(3):414-6. Doi. 10.1096/fj.010711fje.

6. Ott H, Baron JM, Heise R, Ocklenburg C, Stanzel S, Merk HF, et al. Clinical usefulness of microarray-based IgE detection in children with suspected food allergy. Allergy. 2008;63(11):1521-8. Doi: 10.1111/j.1398-9995.2008.01748.x.

7. Martínez-Aranguren R, Lizaso MT, Goikoetxea MJ, García BE, CabreraFreitag P, Trellez O, et al. Is the Determination of Specific IgE against components using ISAC 112 a reproducible technique? PLoS One. 2014;9(2):e88394. Doi: 10.1371/journal.pone.0088394.

8. Moreira PF, Gangl K, Vieira Fde A, Ynoue LH, Linhart B, Flicker S, et al. Allergen microarray Indicates Pooideae sensitization in Brazilian grass pollen allergic patients. PLoS one. 2015;10(6):e0128402. Doi: 10.1371/journal.pone.0128402.

9. Robbins MA, Li M, Leung I, Li H, Boyer DV, Song Y, et al., Stable expression of shRNAs in human CD34+ progenitor cells can avoid induction of interferon responses to siRNAs in vitro. Nature biotech. 2006;24(5):566-571. Doi:10.1038/nbt1206.

10. Matson RS. Multiplex immunoassay for allergens using oligonucleotide tethering. $15^{\text {th }}$ Int'l Congress of Immunology; 2013; Milan, Italy;2769-P; doi: 10.3252/pso.eu.15ici.2013. 